\title{
O estado da arte sobre a temática empreendedorismo
}

\section{State of the art on the theme entrepreneurship}

\author{
Luciana Aparecida Barbieri da Rosa ${ }^{1}$ \\ ClandiaMaffini Gomes ${ }^{2}$ \\ Ana Paula Perlin ${ }^{3}$ \\ Francies Diego Motke ${ }^{4}$ \\ Kamila Frizzo 5
}

\section{Resumo}

Este artigo analisou as características das publicações relacionadas ao tema empreendedorismo. A pesquisa foi realizada na base de dados Web of Science da ISI Web of Knowledge, procurando identificar as principais áreas temáticas, autores, tipos de documentos, título das fontes, ano das publicações, instituições, idiomas e países dessas publicações, assim como a identificação dos hot topics e as publicações mais citadas, com os autores que mais publicam na temática pesquisada. A análise dos dados teve por base os cálculos dos índices $h-b$ e $m$ de Banks (2006). De acordo com os resultados deste estudo, constatou-se que a quantidade de artigos publicados no período compreendido entre 2005 e 2014 aumentou gradativamente ao longo do período analisado, concentrando-se nos Estados Unidos a liderança das publicações escritas no idioma inglês, tendo como principais fontes os periódicos Small Business Economics, Journal of Business Venturing e Entrepreneurship Theory and Practice. Dentre os 20 tópicos combinados com o tema pesquisado Entrepreneurship (Empreendedorismo) destacaram-se como hot topics os temas estratégia, inovação, redes, negócios sociais. Nesse sentido, denota-se que a temática envolvendo o empreendedorismo é de grande relevância, tendo grandes desafios no que se refere ao desenvolvimento de estudos que contemplem, principalmente, as áreas de gestão, inovação, aspectos sociais e ambientais.

1 PPGA/UFSM - Doutoranda em Administração - Brasil - E-mail: lucianaaparecidabarbieri@yahoo. com.br

2 Professora Doutora de Administração/UFSM - Brasil - E-mail: clandiamg@gmail.com

3 PPGA/UFSM - Mestranda em Administração - Brasil - E-mail: anapaula.perlin@yahoo.com.br

4 PPGA/UFSM - Mestrando em Administração - Brasil - E-mail: fdmotke@gmail.com

5 Graduação em Administração, Universidade Federal de Santa Maria, UFSM - Brasil - E-mail: kamila.frizzo@gmail.com 
Palavras-chave: Empreendedorismo. Pesquisa bibliométrica. Web of Science.

\section{Abstract}

This article analyzed the characteristics of publications related to the topic Entrepreneurship. The research was conducted in the database Web of Science ISI Web of Knowledge, trying to identify the main subject areas, authors, types of documents, title of the sources, year of publications, institutions, languages and countries these publications, as well as the identification the "hot topics" and the most cited publications with authors who publish in the researched topic. Data analysis was based on the index calculations $h-b$ and $m$ of Banks (2006). According to the results of this study, it was found that the number of articles published in the period between 2005 and 2014, increased gradually over the period analyzed, focusing on the United States to lead the publications written in English with the main source the journal Small Business Economics, Journal of Business Venturing and Entrepreneurship Theory and Practice. Among the 20 topics combined with the theme researched Entrepreneurship stood out as "hot topics" the Strategy themes, Innovation, Networking, Social Business. In this sense it denotes that the issue involving the Entrepreneurship is of great importance, with major challenges as regards the development of studies that address primarily the areas of management, innovation, social and environmental aspects.

Keywords: Entrepreneurship. Bibliometric research. Web of Science.

\section{Introdução}

O empreendedorismo tem um papel fundamental na criação e no desenvolvimento dos negócios, estando estreitamente relacionado com o crescimento e prosperidade das nações e regiões. De acordo com Hisrich et al. (2014), o empreendedorismo envolve uma ação empreendedora por meio da criação de novos produtos/processos e/ ou entrada em novos mercados, o que pode ocorrer com a atuação de uma organização recém-criada, ou mesmo dentro de uma organização já estabelecida. Em outras palavras, para McMullen e Shepherd (2006), ser um empreendedor significa agir diante de uma oportunidade na qual se julga valer a pena investir. Conforme Shane e Venkataraman (2000), oportunidades empreendedoras são caracterizadas por situações nas quais novos bens, serviços, matérias-primas e métodos organizacionais 
podem ser introduzidos e vendidos por um valor superior ao seu custo de produção.

As pesquisas na temática do empreendedorismo têm contribuições significativas, em seu desenvolvimento inicial, das áreas de Economia e Psicologia. Entre os economistas, Schumpeter (1985) se destaca entre os pioneiros a verificar e ressaltar a importância do empreendedor na economia, representado pelo agente que implementa inovações, assumindo os riscos de suas ações, tendo o lucro como seu objetivo. Dessa forma, para o autor, o empreendedorismo é o "motor da economia capitalista", na medida em que a geração de novos produtos e a busca de novos mercados é capaz de promover o desenvolvimento socioeconômico (SCHUMPETER, 1985).

Nessa perspectiva, Wiklund et al. (2011) complementam que o empreendedorismo consiste em um tema emergente que vem sendo cada vez mais discutido no âmbito acadêmico, sendo uma área com grande crescimento nos últimos anos. Tendo em vista a importância do empreendedorismo no desenvolvimento econômico, bem como sua relevância no crescimento das organizações, essa temática vem sendo amplamente explorada no âmbito acadêmico. São diversas as iniciativas de compreender o comportamento empreendedor, as características associadas ao sucesso, as atitudes e tolerância frente aos riscos envolvidos, a tomada de decisão na ação empreendedora, os impactos do empreendedorismo no desenvolvimento econômico e empresarial, entre outras diversas abordagens de pesquisa. Partindo da premissa de que é um tema emergente e ainda são poucos os estudos buscando ampliar o conhecimento referente à produção científica relacionada à esta temática, este artigo busca entender quais as características da produção científica internacional sobre o empreendedorismo na última década.

Nesse sentido, foi desenvolvido um estudo bibliométrico, por meio da base de dados Web of Science, considerando as publicações relacionadas ao tema entre os anos de 2004 e 2014. Com isso, o objetivo é identificar as principais características da produção científica 
internacional relacionada ao empreendedorismo na última década. A justificativa para a escolha temporal de pesquisa nos últimos dez anos tem como intuito compreender a evolução da temática, bem como sua importância para o desenvolvimento econômico mundial.

Para alcançar o objetivo proposto, este artigo está estruturado em cinco seções, incluindo esta. A segunda apresenta a contextualização da temática do empreendedorismo no âmbito acadêmico, com o intuito de dar aporte teórico ao estudo, incluindo outros estudos bibliométricos já realizados sobre o tema. A seção seguinte apresenta o método do estudo, em que são descritos os procedimentos adotados no desenvolvimento desta pesquisa. Na sequência, é realizada a análise e a discussão dos resultados obtidos; e, por fim, são apresentadas as considerações finais do estudo.

\section{Referencial teórico}

Esta seção aborda uma revisão na literatura sobre os conceitos de empreendedorismo e sua evolução.

\subsection{Empreendedorismo}

O empreendedorismo há muito tempo está presente no nosso entorno, seja no âmbito acadêmico, empresarial ou pessoal, pois se apresenta como estimulador de quebras de paradigmas na sociedade. Porém, nos últimos tempos, o estudo sobre empreendedorismo vem se intensificando, resultado do avanço tecnológico, da sofisticação da economia e dos meios de produção e serviços, tornando o papel do empreendedor cada vez mais fundamental na sociedade (DORNELLAS, 2014).

Baú (2015) também evidencia que o empreendedorismo ganhou dimensão global por causa das mudanças nas relações internacionais entre nações e empresas, entre o modo de produção, os mercados de trabalho e a formação profissional. Dessa forma, percebe-se que o empreendedorismo tem uma função importante na criação e no 
crescimento dos negócios, assim como no crescimento e na prosperidade de nações e regiões (HISRICH; PETERS; SHEPHERD, 2014).

O empreendedorismo busca compreender de que forma surgem as oportunidades para a criação de produtos, serviços, mercados e processos de produção inovadores, além de entender como são organizadas essas formas e as novas tecnologias e como são descobertas por indivíduos para produzir grandes resultados (BARON; SHANE, 2007). Assim, o empreendedorismo se apresenta como atividade fundamental para a geração de riquezas, por meio do crescimento econômico e do aprimoramento das condições de vida da população, sendo também importante fator de geração de emprego e renda (GEM, 2010), evidenciando a sua importância e contribuição para o desenvolvimento de um país mais sustentável.

Complementando, Costa, Cericato e Melo (2007) afirmam que o empreendedorismo consiste na criação de valor por pessoas e organizações trabalhando juntas para implementar uma ideia por meio da criatividade e capacidade de transformação. Desse modo, o empreendedorismo possui como característica inerente a inovação, e essas temáticas devem andar de mãos dadas, pois assim as múltiplas dimensões de relação da empresa com seu ambiente (desenvolvimento institucional, de recursos alocação e comercialização) permitirão a inovação se desenvolver (WOOLLEY; ROTTNER 2008).

Acredita-se que a sociedade está na era do empreendedorismo, pois os empreendedores estão eliminando barreiras comerciais e culturais, encurtando distâncias, globalizando e renovando os conceitos econômicos, criando novas relações de trabalho e novos empregos, quebrando paradigmas e gerando riqueza para a sociedade (DORNELLAS, 2014).

\section{2 Evolução do empreendedorismo}

De acordo com Zen e Fracasso (2008), o termo empreendedor vem acompanhando as mudanças no contexto social e tecnológico, possuindo três momentos importantes na história. 
O primeiro acontecimento se refere à Revolução Industrial Britânica de 1770, no qual o empreendedor cumpria o papel de assumir riscos e enfrentar incertezas, na expectativa de obter lucro. Após, temse a implantação do modelo de produção fordista nos Estados Unidos da América (EUA), em 1900, quando o termo "empreendedor" adquiriu uma nova abordagem, relacionada à imaginação e inovação. Por fim, constata-se que, a partir da emergência das tecnologias da informação, nos anos 1990, houve uma expansão das possibilidades de aplicar o conhecimento no desenvolvimento de novos serviços e produtos, desenvolvidos por milhares de novas empresas.

De acordo com Falcone e Osborne (2005), muitos estudiosos tentam apresentar uma definição do empreendedorismo, porém, apresentam visões, pensamentos e comportamentos diferentes.

Para Kirzner (1973), o empreendedor é aquele que cria um equilíbrio, encontrando uma posição clara e positiva em um ambiente de caos e turbulência. Além disso, caracteriza-se como empreendedora a pessoa que identifica clientes potenciais e cria uma empresa como oportunidade de negócios para satisfazê-lo (MARCONDES 2000). Conceitua-se também como empreendedor aquele que detecta uma oportunidade e cria um negócio para capitalizar sobre ela, assumindo riscos calculados (DORNELAS, 2001).

Para Hisrich, Peters e Shepherd (2014), ser empreendedor significa agir diante de uma oportunidade que vale a pena ser trabalhada, ou seja, ato empreendedor é algo que as pessoas optam por fazer com base nas percepções da predisposição e viabilidade de criar um novo empreendimento para realizar uma oportunidade.

Até a primeira metade do século $\mathrm{XX}$, as empresas surgiam e se expandiam sem grandes preocupações com os impactos ambientais e sociais. Dessa forma, vem sendo muito explorado no âmbito acadêmico o termo "empreendedorismo social e sustentável". O interesse no empreendedorismo social tem aumentado nos últimos anos, como evidenciado pelos números de conferências, edições especiais e artigos 
de pesquisa que aparecem em uma ampla gama de publicações (MOSS et al., 2011).

Segundo Tidd e Bessant (2009), os empreendedores sociais percebem um problema social e utilizam os princípios empreendedores tradicionais para organizar, criar e administrar um empreendimento visando realizar mudança social. Esse empreendedor reúne esforços para incorporar práticas de mercado com o desenvolvimento humano, oferecendo soluções para os problemas sociais, como o combate à pobreza e a incorporação dos excluídos aos direitos básicos de cidadania (MELO NETO; FROES, 2002).

Além disso, o empreendedorismo social carrega a inovação como um componente essencial de sua definição, sendo transformador de uma realidade local, nacional ou mundial (SOCIALEDGE, 2008).

O empreendedorismo sustentável, segundo Hockerts e Wüstenhagen (2003), consiste na identificação de uma oportunidade de inovação sustentável e sua implementação, seja por meio de um novo negócio ou reorientação radical do negócio existente.

Entende-se, ainda, que o empreendedor "verde" é uma classificação que se diferencia de outras, porque objetiva, em geral, uma sustentabilidade entre o uso de recursos e negócios (AVENI, 2014).

Da mesma forma, o empreendedorismo sustentável pode ser definido como a descoberta, o desenvolvimento e a exploração de oportunidades ligadas aos nichos sociais e ambientais que geram ganho econômico e melhoria social e ambiental.

O termo "empreendedorismo" também vem sendo muito abordado na temática de inovação. O empreendedorismo inovador consiste em qualquer empreendimento que gere inovação relevante em benefícios do negócio e da sociedade (DRUCKER, 2007), como incubadoras, parques e polos tecnológicos.

Além disso, outro termo que vem sendo relacionado ao empreendedorismo é o setor público. De acordo com Roberts (1992), 
empreendedorismo no setor público é a geração de ideia inovadora, a concepção e a implementação dessa ideia no setor público.

\section{Método}

Este estudo caracteriza-se como exploratório e quantitativo, tendo sido operacionalizado a partir de uma pesquisa bibliométrica, objetivando ampliar o conhecimento referente às publicações relacionadas ao tema empreendedorismo (entrepreneurship) na base de dados Web of Science, no período compreendido entre os anos de 2005 e 2014.

Segundo Guedes e Borschiver (2005, p. 15), a bibliometria "possibilita mapear e gerar indicadores de tratamento e gestão da informação e do conhecimento necessários ao planejamento, avaliação e gestão da ciência e da tecnologia, de uma determinada comunidade científica ou país".

\subsection{Definição do escopo do estudo}

A busca das publicações para fins de realização da bibliometria foi realizada a partir da base de dados da Web of Science (WOS) do Institute for Scientific Information (ISI).

A WOS consiste em uma base multidisciplinar que possui cerca de 9.000 periódicos indexados. A base indexa somente os periódicos mais citados em suas respectivas áreas. É também um índice de citações, informando, para cada artigo, os documentos por ele citados e os documentos que o citaram. Permite, ainda, analisar as características das publicações e o cálculo de índices de citações (CAPES, 2015).

Foi pesquisado o termo entrepreneur* na principal coleção da Web of Science, no período compreendido entre os anos de 2005 e 2014, sendo encontrados 19.297 trabalhos. Posteriormente, refinou-se a busca para publicações das categorias management e business, e para a exibição apenas de artigos, resultando em 5.501 trabalhos. 


\subsection{Etapas para a coleta dos dados}

A realização da pesquisa dividiu-se em quatro etapas. Inicialmente digitou-se o termo entrepreneur ${ }^{\star}$ no campo de pesquisa da base WOS. Adotou-se o asterisco para contemplar termos com diferentes terminações relacionados à palavra "empreendedorismo" eEntrepreneurship), delimitando-se o período de 2005 a 2014. A seguir, foram levantadas as características gerais das publicações: principais autores, principais países e idiomas, ano das publicações, título das fontes, principais instituições.

$\mathrm{Na}$ segunda etapa, foram identificados os principais tópicos relacionados à temática empreendedorismo. Com base em uma análise prévia das publicações encontradas na Web of Science, foram selecionados 20 tópicos relacionados ao tema.

$\mathrm{Na}$ terceira etapa, realizou-se a combinação de cada tópico com o termo entrepreneur", sendo calculado o total de publicações para cada combinação (tópico relacionado), o $h$-index e o coeficiente $m$.

Em seguida, na quarta etapa, foram identificados os hot topics por meio do cálculo do índice $h$-b e $m$.

\subsection{Identificação dos hot topics}

Inicialmente, foram analisados os índices $h-b$ e $m$ para posteriormente realizar-se a análise dos hot topics. Hirsch (2005) propõe o índice $h$-index (índice-h), visando quantificar o impacto e a relevância da produção científica individual para a avaliação de pesquisadores e comparação de propósitos de pesquisa.

Banks (2006) propõe o índice $h$-b como uma extensão do $h$-index, que é obtido por meio do número de citações de um tópico ou combinação em determinado período, listados em ordem decrescente de citações. $O$ índice $h$ - $b$ é encontrado em publicações que tenham obtido um número de citações igual ou maior à sua posição no ranking. Banks (2006) também explica o cálculo do índice $m$, o qual é obtido pela divisão do 
índice $h-b$ pelo período de anos que se deseja obter informações $(n)$. Para a análise dos índices $h-b$ e $m$, foram utilizadas as definições de Banks (2006), evidenciadas no Quadro 1.

Quadro 1 - Definições para classificação de hot topics

\begin{tabular}{|l|l|}
\hline Índicem & \multicolumn{1}{c|}{ Tópico/combinação } \\
\hline $0<\mathrm{m} \leq 0,5$ & $\begin{array}{l}\text { Pode ser de interesse para pesquisadores em um campo } \\
\text { específico de pesquisa, o qual engloba uma comunidade } \\
\text { pequena. }\end{array}$ \\
\hline $0,5<\mathrm{m} \leq 2$ & $\begin{array}{l}\text { Provavelmente pode se tornar um hot topic como área de } \\
\text { pesquisa, no qual a comunidade é muito grande ou o tópico/ } \\
\text { combinação apresenta características muito interessantes. }\end{array}$ \\
\hline $\mathrm{m} \geq 2$ & $\begin{array}{l}\text { É considerado um hot topic, tema exclusivo com alcance } \\
\text { não apenas na sua própria área de pesquisa e é provável } \\
\text { que tenha efeitos de aplicação ou características únicas. }\end{array}$ \\
\hline
\end{tabular}

Fonte: Banks (2006).

A partir das definições de Banks (2006), neste estudo, serão considerados hot topics as combinações com índice $m \geq 2$.

\section{Análise e discussão dos resultados}

Realizou-se a pesquisa do termo entrepreneur* na principal coleção da Web of Science, no período compreendido entre os anos de 2005 e 2014, e foram encontrados 19.297 trabalhos. Após refinar a busca para publicações das categorias management e business, e para a exibição apenas de artigos, a pesquisa resultou em 5.501 trabalhos. Inicialmente, serão apresentados os resultados gerais das publicações e, por fim, os hot topics relacionados ao tema.

\subsection{Características gerais das publicações}

A seguir, serão apresentadas as características gerais das publicações relacionadas ao tema empreendedorismo, de acordo com 
as seguintes categorias: autores, países e idioma das publicações, ano das publicações, título das fontes e principais instituições.

\subsubsection{Principais autores}

O Quadro 2 apresenta os principais autores que publicaram sobre o tema empreendedorismo nos últimos dez anos.

Quadro 2 - Principais autores

\begin{tabular}{|l|c|}
\hline Autor & $\mathbf{N}^{\circ}$ Artigos \\
\hline WRIGHT, M. & 70 \\
\hline SHEPHERD, D. A. & 37 \\
\hline AUDRETSCH, D. B. & 36 \\
\hline ZAHRA, S. A. & 35 \\
\hline URBANO, D. & 24 \\
\hline ACS, Z. J. & 23 \\
\hline LI, Y. & 23 \\
\hline BARON, R. A. & 21 \\
\hline BRUTON, G. D. & 21 \\
\hline DE CLERCQ, D. & 21 \\
\hline IRELAND, R. D. & 21 \\
\hline KURATKO, D. F. & 21 \\
\hline
\end{tabular}

Fonte: Web of Science (2015).

Percebe-se, pelo Quadro 2, que existe uma diversidade de autores que publicaram uma elevada quantidade de trabalhos sobre o tema no período analisado. Além disso, Mike Wright, professor da Imperial College London, Londres, Inglaterra, destaca-se como o pesquisador com maior número de publicações no tema de empreendedorismo nos últimos dez anos. Outros pesquisadores com elevado número de trabalhos no tema são: Dean A. Shepherd e David B. Audretsch, da Indiana University, Estados Unidos; e Shaker A. Zahra, da Universityof Minnesota, Estados Unidos. 


\subsubsection{Principais países e idiomas}

No Quadro 3, apresentam-se os principais países que possuem publicações relacionadas ao tema pesquisado.

Quadro 3 - Principais países

\begin{tabular}{|c|c|c|}
\hline & País & $\mathbf{N}^{\circ}$ Artigos \\
\hline $1^{\circ}$ & Estados Unidos & 2186 \\
\hline $2^{\circ}$ & Inglaterra & 863 \\
\hline $3^{\circ}$ & Canadá & 426 \\
\hline $4^{\circ}$ & Espanha & 414 \\
\hline $5^{\circ}$ & Alemanha & 381 \\
\hline $6^{\circ}$ & Holanda & 334 \\
\hline $7^{\circ}$ & China & 254 \\
\hline $8^{\circ}$ & Suécia & 243 \\
\hline $9^{\circ}$ & Austrália & 222 \\
\hline $10^{\circ}$ & Itália & 206 \\
\hline $22^{\circ}$ & Portugal & 58 \\
\hline $23^{\circ}$ & Brasil & 32 \\
\hline
\end{tabular}

Fonte: Web of Science (2015).

No que diz respeito ao número de publicações por países, os Estados Unidos da América lideram o ranking, seguidos de Inglaterra, Canadá e Espanha. Com isso, percebe-se que os países com mais publicações são os países dos principais pesquisadores da temática empreendedorismo. Destaca-se que Portugal ocupa a $22^{a}$ posição e o Brasil ocupa a $23^{a}$, o que revela uma grande disparidade do número de publicações destes países em relação aos que estão no topo.

Corroborando com os países que mais publicam sobre a temática, o idioma inglês se sobressai, com 5.418 publicações, ou seja, 98,5\% do total de estudos publicados no tema. 


\subsubsection{Ano das publicações}

No período compreendido entre 2005 e 2014, constatou-se que o número de publicações aumentou gradativamente ao longo do período analisado. A Figura 1 apresenta a quantidade de artigos publicados por ano relacionado ao tema Empreendedorismo.

Figura 1 - Publicações por ano

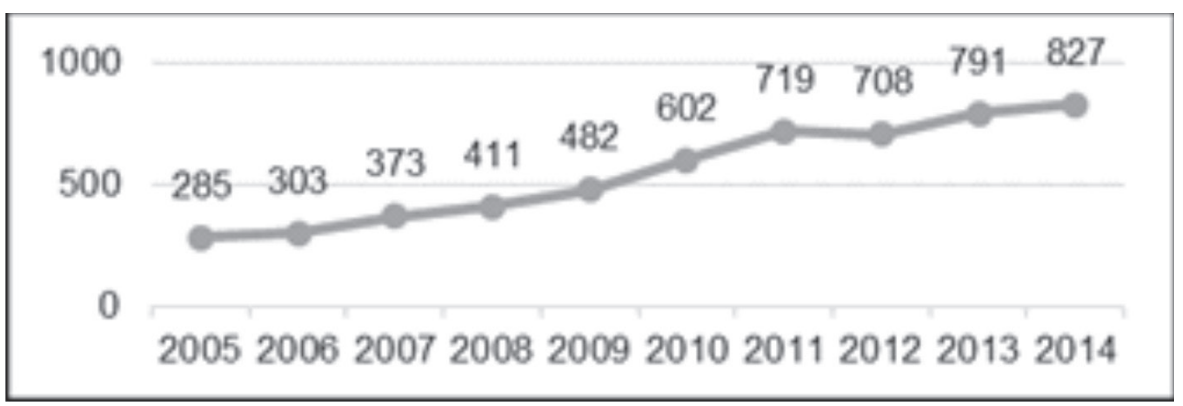

Fonte: Web of Science (2015).

Comparando o número de publicações do ano 2005 a 2014, evidencia-se que a houve um crescimento significativo (quase triplicado) no período, demonstrando a relevância que os estudos sobre empreendedorismo estão ganhando nos últimos anos.

\subsubsection{Título das fontes}

O Quadro 4 apresenta as dez principais fontes de publicações relacionadas à temática empreendedorismo. 
Quadro 4 - Título das fontes

\begin{tabular}{|l|c|}
\hline Título da Fonte & No Artigos $^{\circ}$ \\
\hline Small Business Economics & 396 \\
\hline Journal of Business Venturing & 327 \\
\hline Entrepreneurship Theory and Practice & 322 \\
\hline Entrepreneurship and Regional Development & 217 \\
\hline International Small Business Journal & 183 \\
\hline Research Policy & 159 \\
\hline Journal of Small Business Management & 154 \\
\hline International Entrepreneurship and Management Journal & 143 \\
\hline Strategic Entrepreneurship Journal & 124 \\
\hline Technovation & 120 \\
\hline
\end{tabular}

Fonte: Web of Science (2015).

Os periódicos que mais têm publicado trabalhos na temática são: Small Business Economics, Journalof Business Venturing e Entrepreneurship Theory and Practice. Percebe-se que os dois periódicos que mais publicam estudos no tema empreendedorismo não tratam especificamente desse tema. Destaca-se que entre os dez principais periódicos que têm publicado sobre empreendedorismo quatro tratam especificamente da temática e outros três são direcionados aos pequenos negócios.

\subsubsection{Principais instituições}

As instituições que mais publicaram trabalhos relacionados ao tema empreendedorismo estão em destaque no Quadro 5. 
Quadro 5 - Principais instituições

\begin{tabular}{|l|c|}
\hline Instituição & $\mathbf{N}^{\circ}$ Artigos \\
\hline Indiana University & 157 \\
\hline University of Nottingham & 94 \\
\hline Erasmus University & 82 \\
\hline University of Minnesota & 71 \\
\hline University of North Carolina & 70 \\
\hline University of Illinois & 67 \\
\hline Imperial College London & 67 \\
\hline Babson College & 65 \\
\hline Universitat de València & 65 \\
\hline Harvard University & 63 \\
\hline
\end{tabular}

Fonte: Web of Science (2015).

As instituições que mais se destacaram no que se refere às publicações relacionadas ao tema empreendedorismo foram: Indiana University, dos Estados Unidos, University of Nottingham, da Inglaterra, e Erasmus University, da Holanda.

\subsubsection{Empreendedorismo e os hot topics}

Nesta etapa da pesquisa, foram investigadas as publicações sobre empreendedorismo e os principais tópicos relacionados a essa temática. Com base em uma análise prévia das publicações encontradas na Web of Science, foram selecionados 20 tópicos relacionados ao tema.

O Quadro 6 classifica os 20 tópicos relacionados à temática, organizados conforme o número de publicações após a seleção das categorias management e business, e da opção de exibição apenas de artigos. 
Quadro 6 - Tópicos relacionados ao tema empreendedorismo (20052014)

\begin{tabular}{|l|c|}
\hline Tópicos & $\begin{array}{c}\text { Total de } \\
\text { Publicações }\end{array}$ \\
\hline Estratégia (Strategy) & 18.820 \\
\hline Inovação (Innovation) & 12.918 \\
\hline Redes (Networks) & 10.305 \\
\hline Cultura (Culture) & 5.929 \\
\hline Conhecimento organizacional (Organizational knowledge) & 4.483 \\
\hline Governo (Government) & 4.209 \\
\hline Negócios sociais (Social business) & 4.002 \\
\hline Ética (Ethics) & 3.075 \\
\hline Responsabilidade social (Social responsibility) & 2.897 \\
\hline Fracasso (Failure) & 2.801 \\
\hline Gestão ambiental (Environmental management) & 2.066 \\
\hline Setor público (Sector public) & 1.669 \\
\hline Internacionalização (Internationalization) & 1.421 \\
\hline Impostos (Taxes) & 781 \\
\hline Fundos de investimento (Investment funds) & 527 \\
\hline Franquia (Franchise) & 298 \\
\hline Startup & 145 \\
\hline Incubadora (Incubator) & 145 \\
\hline Base da pirâmide (Bottom of the pyramid) & 74 \\
\hline Parques tecnológicos (Technological park) & 26 \\
\hline
\end{tabular}

Fonte: Web of Science (2015).

Posteriormente, realizou-se a combinação de cada tópico com o termo entrepreneur ${ }^{*}$, sendo calculado o total de publicações para cada combinação (tópico relacionado), o $h$-index e o coeficiente $m$, apresentada no Quadro 7. 
Quadro 7 - Hot topics relacionados ao tema empreendedorismo

\begin{tabular}{|c|c|c|c|}
\hline Tópicos & $\begin{array}{c}\text { Total de } \\
\text { Publicações }\end{array}$ & $\begin{array}{l}\text { Índice } \\
\text { H-B }\end{array}$ & Índice M \\
\hline Estratégia (Strategy) & 1291 & 65 & 6,5 \\
\hline Inovação (Innovation) & 1734 & 63 & 6,3 \\
\hline Redes (Networks) & 997 & 52 & 5,2 \\
\hline Negócios sociais (Social business) & 613 & 40 & 4,0 \\
\hline $\begin{array}{l}\text { Conhecimento organizacional } \\
\text { (Organizational knowledge) }\end{array}$ & 342 & 37 & 3,7 \\
\hline Internacionalização (Internationalization) & 300 & 37 & 3,7 \\
\hline Cultura (Culture) & 364 & 30 & 3,0 \\
\hline Fracasso (Failure) & 246 & 30 & 3,0 \\
\hline Governo (Government) & 361 & 29 & 2,9 \\
\hline $\begin{array}{l}\text { Responsabilidade social } \quad \text { (Social } \\
\text { responsibility) }\end{array}$ & 149 & 25 & 2,5 \\
\hline Startup & 91 & 22 & 2,2 \\
\hline $\begin{array}{l}\text { Gestão ambiental (Environmental } \\
\text { management) }\end{array}$ & 138 & 21 & 2,1 \\
\hline Incubadora (Incubator) & 78 & 19 & 1,9 \\
\hline Ética (Ethics) & 120 & 17 & 1,7 \\
\hline Setor público (Sector public) & 103 & 16 & 1,6 \\
\hline $\begin{array}{l}\text { Fundos de investimento (Investment } \\
\text { funds) }\end{array}$ & 89 & 13 & 1,3 \\
\hline Franquia (Franchise) & 53 & 12 & 1,2 \\
\hline Impostos (Taxes) & 61 & 12 & 1,2 \\
\hline Base da pirâmide (Bottom of the pyramid) & 19 & 6 & 0,6 \\
\hline $\begin{array}{l}\text { Parques tecnológicos (Technological } \\
\text { park) }\end{array}$ & 8 & 6 & 0,6 \\
\hline
\end{tabular}

Fonte: Web of Science (2015).

De acordo com Kelly e Jannions (2006), a partir do cálculo dos índices $h$-index e $m$, é possível mensurar o desempenho dos tópicos/ combinações pesquisados, tendo por base o número de citações que tiveram. Baseando-se nas considerações de Banks (2006), pode-se 
classificar como hot topics as combinações do tema empreendedorismo com os doze tópicos classificados no Quadro 7: estratégia (strategy), inovação (innovation), redes (networks), negócios sociais (social business), conhecimento organizacional (organizational knowledge), internacionalização (internationalization), cultura (culture), fracasso (failure), governo (government), responsabilidade social (social responsibility), startup e gestão ambiental (environmental management).

Desse modo, percebe-se que esses doze tópicos relacionados com a temática empreendedorismo são hot topics por apresentarem $m \geq$ 2 , podendo ser classificados como tópicos exclusivos, com alcance não apenas na própria área de pesquisa, e provavelmente têm efeitos de aplicação ou características únicas.

As outras combinações que apresentarem índice $0,5<m \leq 2$ podem ser consideradas hot topics emergentes como áreas de pesquisa. Os tópicos que apresentaram $m \leq 0,5$ podem ser de interesse para pesquisadores em um campo específico de pesquisa.

Nesse sentido, denota-se que a temática envolvendo 0 empreendedorismo é de grande relevância, tendo grandes desafios no que se refere ao desenvolvimento de estudos que contemplem, principalmente, as áreas de gestão, inovação, aspectos sociais e ambientais.

\section{Considerações finais}

A análise das publicações sobre entrepreneurship na base de dados Web of Science resultou em 5.501 artigos relacionados aos temas management e business (administração e gestão). Constatou-se que o número de artigos publicados no período compreendido entre 2005 e 2014 aumentou gradativamente ao longo do período analisado.

A maioria das publicações referente ao tema foi publicada nos periódicos Small Business Economics, Journal of Business Venturing e Entrepreneurship Theory and Practice, que se destacam com o maior número de publicações. As instituições que mais publicaram artigos 
relacionados ao tema foram Indiana University, Universityof Nottingham e Erasmus University. Verificou-se, ainda, que os Estados Unidos da América (EUA) lideram o ranking dos países que mais publicaram sobre a temática.

A combinação do tópico entrepreneurship (empreendedorismo) com os 20 tópicos resultou em 12 combinações que apresentaram índice $m \geq 2$, podendo ser consideradas hot topics emergentes nas seguintes áreas de pesquisa :estratégia, inovação, redes, negócios sociais, conhecimento organizacional, internacionalização, cultura, fracasso, governo, responsabilidade social, startup e gestão ambiental.

Como limitação do estudo, destaca-se a sua realização utilizando apenas em uma base de dados específica. Como sugestão para trabalhos futuros, pode-se ampliar o escopo dos meios de publicação, contemplando outras importantes bases de dados científicos, anais de eventos acadêmicos nacionais e internacionais, e periódicos classificados como 'A' no Sistema Qualis /CAPES.

\section{Referências}

AVENI, A. Empreendedorismo contemporâneo: teorias e tipologias. São Paulo: Atlas, 2014.

BANKS, M. G. An extension of the Hirsch index: indexing scientific topics and compounds. Scientometrics, Budapeste, Hungria, v. 69, n. 1, p. 161-168, Oct. 2006. Disponível em: <http://sci2s.ugr.es/sites/ default/files/files/TematicWebSites/hindex/Banks2006.pdf>. Acesso em: 15 Jun. 2015.

BAÚ, E. C. O empreendedorismo e os pequenos negócios como alternativa de desenvolvimento sustentável para Roraima. 2011. 103f. Dissertação (Mestrado em Econmia) - Universidade Federal do Rio Grande do Sul, Faculdade de Ciências Econômicas, Porto Alegre, 2011. Disponível em: <http://www.lume.ufrgs.br/bitstream/ handle/10183/40248/000822336.pdf?sequence=1> Acesso em: 18 ago. 2015. 
BESSANT, J; TIDD, J. Inovação e empreendedorismo. Porto Alegre: Bookman, 2009.

COORDENAÇÃO DE APERFEIÇOAMENTO DE PESSOAL DE NÍVEL SUPERIOR (CPES). Acervo 201. Disponível em: < http://periodicos. capes.gov.br.ez47.periodicos.capes.gov.br/index.php?option=com _ pcollection\&ltemid=104>. Acesso em: 16 jul. 2015.

DORNELAS, J. C. A. Empreendedorismo: transformando ideias em negócios. 5 ed. Rio de Janeiro: Empreende, 2014.

GUEDES, V. L.S.; BORSCHIVER, S. Bibliometria: uma ferramenta estatística para a gestão da informação e do conhecimento, em sistemas de informação, de comunicação e de avaliação científica e tecnológica. In: ENCONTRO NACIONAL DE CIÊNCIAS DA INFORMAÇÃO, 6., 2005, Salvador. Anais... Salvador/BA, Brasil: CINFORM, 2005. p. 1-18. Disponivel em: <http://www.cinformanteriores.ufba.br/vi_anais/docs/VaniaLSGuedes.pdf>. Acesso em 16 jul. 2015.

HISRICH, R. D.; PETERS, M. P.; SHEPHERD, D. A. Empreendedorismo. 9. ed. Porto Alegre: AMDH, 2014.

HOCKERTS, K.; WÜSTENHAGEN, R. Greening Goliaths versus emeying Davids. 2. ed. Frederiksberg: Center for Corporate Social Responsibility (CBS), 2003.

KELLY, C. D.; JENNIONS, M. D. The $\mathrm{h}$ index and career assessment by numbers. Trends in Ecology and Evolution, Cambridge, MA, v. 21, n. 4, p. 167-170, Abr., 2006.

KIRZNER, I. M. Entrepreneurial discovery and the competitive market process: an austrian approach, Journal of Economic Literature, Pittsburgh, PA, v. 35, n. 1, p. 60-85, Mar. 1997.

LUMPKIN, G. T.; MOSS, T. W.; GRAS, D. M.; KATO S.; AMEZCUA, A. S. Entrepreneurial processes in social contexts: how are they different, if at all?. Germany: Springer Science + Business Media: LLC. 2011. 
MCMULLEN, J. S.; D. A. SHEPHERD, D. A. Entrepreneurial action and the role of uncertainty in the theory of the entrepreneur. The Academy of Management Review, Newton, MA, v. 31, n1, p. 132-152, Jan. 2006.

SCHUMPETER, J. A. Teoria do desenvolvimento econômico: uma investigação sobre lucros, capital, crédito, juro e o ciclo econômico. 2. ed. São Paulo: Nova Cultural, 1985.

SHANE, S.; VENKATARAMAN, S. The promise of entrepreneurship as a field of research. The Academy of Management Review, Newton, MA, v. 25, n. 1. p. 217-226, Jan. 2000.

SOCIALEDGE. By social entrepreneurship for social

entrepreneurship. 2008. Disponível em: <http://skollworldforum.org/ category/social-edge/>. Acesso em: 02 ago. 2015.

ZEN, A. C.; FRACASSO, E. M. Quem é o empreendedor? As implicações de três revoluções tecnológicas na construção do termo empreendedor. RAM - Revista de Administração de Mackenzie, São Paulo, v. 9, n. 8, Edição Especial, p. 135-150. nov./dez. 2008.

Artigo recebido em: 21/09/2015 Aprovado em: 18/12/2015 